\title{
Psychomotor therapy for anger and aggression in mild intellectual disability or borderline intellectual functioning: an intervention mapping approach
}

Difficulties with anger regulation in individuals with mild intellectual disability or borderline intellectual functioning (MID-BIF) are often associated with aggressive behaviour. Psychomotor therapy (PMT), a mind-body intervention often applied in Dutch facilities for this target group, uses body awareness and physical activities as a medium to learn how to gain control over one's anger and reduce aggressive behaviour. With Intervention Mapping as a framework, 17 Dutch psychomotor therapists were asked to describe their PMT programme to investigate its content and coherence. Results suggested that interoceptive awareness (IA) and adaptive coping skills (ACS) are key elements of the PMT programmes. The theoretical basis of the PMT programmes is however limited and specific instruments for evaluating PMT are not available yet, especially for IA. Psychomotor therapists would gain from a welldescribed PMT programme. Future research is warranted on IA, and specific instruments need to be developed and validated for this target group.

Keywords: psychomotor therapy; mild intellectual disabilities; borderline intellectual functioning; anger; aggressive behaviour; Intervention Mapping 


\section{Introduction}

Psychomotor therapy (PMT), an experiential therapy, is defined as a treatment that uses body awareness and physical activities as cornerstones of its approach. It is based on a holistic view, which integrates cognitive, emotional and motor aspects of an individual's functioning (Probst, Knapen, Poot, \& Vancampfort, 2010). PMT aims to offer 'a playground' for participants to increase their ability to recognize differences in one's own body signals, i.e. 'interoceptive awareness' (IA), and practice with different forms of responding. Through actively participating in exercises, awareness of body signals, emotions and thoughts increases and there is growing recognition of one's own behavioural reactions (see Boerhout, Swart, Busschbach, \& Hoek, 2013; Maas et al., 2015; Röhricht, 2009; Zwets et al., 2016). PMT is well embedded in health care in Europe although the term PMT is not an established term in the English literature. Other terms like Body-oriented Psychotherapy or Dance and Movement Therapy are more commonly used (Probst et al., 2010).

PMT seems to be effective in individuals who experience anger or present with aggressive behaviour (Boerhout et al., 2013; Zwets et al., 2016). In individuals with limited cognitive and verbal abilities aggressive behaviour often occurs, with anger as a potential risk factor (Novaco, 1994; Taylor \& Novaco, 2005). Due to the experiential nature of PMT that relies less on clients' verbal skills, PMT seems feasible as a treatment for individuals with limited cognitive and verbal abilities such as individuals with mild intellectual disabilities (MID, IQ 50-70) or borderline intellectual functioning (BIF, IQ 70-85) (McDonnell et al., 2015; Kay, Clegg, Emck, \& Standen, 2016). As these two groups share many characteristics and needs (namely, deficits in cognitive and adaptive functioning and psychosocial problems), they are merged in both scientific literature and Dutch clinical practice. Therefore, this group is defined as MID-BIF (Wieland \& Zitman, 2015).

A systematic review demonstrated that, based on the criteria of empirically supported therapies (see Chambless \& Ollendick, 2001), PMT is a promising approach in individuals with MIDBIF showing aggressive behaviour (Bellemans et al., in press). However, the review suggested that the 
evidence around PMT currently lacks clarity regarding the process, mechanisms of action, and target outcomes.

The absence of a standardised programme targeting aggressive behaviour as the main problem, with explicit objectives and outcomes, necessary in the context of a transition from practisebased interventions to evidence-based practise, is not uncommon in this field. Although there is a growing interest in evidence-based interventions for individuals with intellectual disabilities scientific research has traditionally been ignored (see e.g., Sturmey \& Didden, 2014). Furthermore, practicebased interventions, such as PMT, or even interventions that have been demonstrated to be ineffective, are frequently applied in clinical practice with this population (Goin-Kochel, Mackintosh, \& Myers, 2009; Goin-Kochel, Myers, \& Mackintosh 2007). To improve the quality of care, individual clinical expertise needs to be integrated with the best available evidence from systematic research (Singh, 2016).

Intervention Mapping has been proposed as a way to systematically develop and / or evaluate theory- and evidence-based programmes (Eldredge et al., 2016; Kok, Bartholomew, Parcel, Gottlieb, \& Fernández, 2014). Intervention Mapping is a six-step framework that integrates evidence from existing research with clinical practice to develop a coherent programme.

In the first step, called logic model of the problem, the problem which is targeted in the programme, is described. In the second step, called logic model of change, the expected and measurable outcomes of the programme are expressed. Programme design, the third step, identifies intervention methods to achieve the outcomes identified in step 2. The rationale for the method selection needs to be based on clear theoretical links between the method and the problem (step 1) as well as existing evidence, i.e., intervention methods that have been shown to produce change. In programme production, the fourth step, the problems, programme outcomes and methods found in the first three steps are brought together to design a coherent intervention programme.

Implementation plan, the fifth step, determines the scaling-up of the programme, and how to sustain implementation in real-life contexts. At this stage, it is crucial to involving both policy makers and practitioners. In the final step, evaluation of the programme, an evaluation plan is made to test whether the expected outcomes, mentioned in the second step, are achieved by the programme (i.e., outcome 
evaluation) as well as a plan for the evaluation of the programme's process (i.e., process evaluation). Completion of these six iterative steps results in a template for implementing and evaluating a programme whose design has integrated theoretical, empirical and practical information.

In the present study we used Intervention Mapping to gather detailed information on the PMT programmes that are currently applied when individuals with MID-BIF have problems managing their own anger and aggressive behaviour. The aim of this study was to describe the theoretical orientation, process content, implementation and evaluation of PMT programmes as used by psychomotor therapists in facilities for individuals with MID-BIF and anger regulation problems or aggressive behaviour. We interviewed 17 psychomotor therapists on how they currently apply PMT, its objectives and outcomes, perceived mechanisms of action, theoretical background, and evaluation. Similar to the method followed by Schaafsma and colleagues (2013) we asked multiple questions per step. The six steps of Intervention Mapping were thus used as a post hoc framework to map the programmes' content as well as their coherence. It was anticipated that interviews would increase our understanding of the way PMT programmes are used in practice, while the Intervention Mapping framework would enable a systematic description of their application and evaluation methods. Developing a standardised PMT programme can be used to study the effectiveness of PMT as an intervention for aggressive behaviour or anger regulation difficulties in individuals with MID-BIF.

\section{Method}

\section{Participants}

For this study, a pre-selected sample of psychomotor therapists who are members of the Dutch Association for PMT [NVPMT; Nederlandse Vereniging voor Psychomotorische Therapie] and who on earlier occasions had expressed an interest in participating in research, were contacted to fill in the questionnaire. Therapists had to have at least three years' experience in the treatment of anger management problems and/or aggressive behaviour with individuals with MID-BIF. In addition, participants were considered only if they had been educated on psychomotor therapy at Master's level, to ensure that they were able to reflect on the intervention and its underlying mechanisms of action. Thirty-six psychomotor therapists were approached individually by e-mail. Thirteen psychomotor therapists did not meet the criteria for inclusion due to the absence of a Master's degree $(n=12)$ or 
only one year of experience with this target group $(n=1)$. Twenty-three therapists met the inclusion criteria. Of those, six did not complete the questionnaire. Seventeen psychomotor therapists (4 males, 13 females) were included in the final analyses. All of them work with individuals with MID-BIF in both inpatient and outpatient settings.

\section{Procedure and Questionnaire Topics}

An online questionnaire, Qualtrics (selection 12.4) [software] (2016), was used to gather information on six topics similar to the steps of the Intervention Mapping (Eldredge et al., 2016). The third author formulated the questions per topic (see Table 1) in line with an earlier adaptation of the Intervention Mapping approach by Schaafsma, Stoffelen, Kok, \& Curfs (2013) and these were discussed and adapted by five experts (one on Intervention Mapping, one on PMT, and three on PMT as applied in ID-facilities).

(insert Table 1)

The psychomotor therapists were informed about the content of the Intervention Mapping approach together with an invitation to participate as they were not familiar with this approach. This gave them the opportunity to prepare in advance, by considering what they do, why they do this, which outcomes they want to achieve and how their PMT-programme is evaluated.

\section{Data Processing and Analysis}

Quantitative data and data of multiple-choice questions were directly analysed in Qualtrics (version 12.4) [software] (2016). All qualitative data were imported in ATLAS.ti (version 7) [software] (2016) for analysis. For each question answers were categorised per topic code (derived from the steps of the Intervention Mapping) by the third author (RV) and the categories were validated by the first author (TB). When discrepancies occurred between the two researchers, the categories were discussed until agreement was reached. Following this, the answers linked to the categories were sent to the psychomotor therapists who could check the answers on correctness and completeness. When needed, additional information was specifically asked for. After receiving feedback from the therapists, the procedure of categorisation, validation and consensus was repeated for the feedback. At the end of this process, a list of categories was developed for each step of the Intervention Mapping, separately for every therapist. This list was once again sent to the psychomotor therapists for a check 
on correctness and completeness. The feedback provided by the therapist was used to review the list, and a final list of categories with answers was created. The information from all psychomotor therapists was then amalgamated. The amalgamation of data from therapists resulted in multiple categories being available under every step of the Intervention Mapping.

\section{Results}

Findings are presented in two sections. First, we provide an overview of the applied PMT programmes, and the various steps of the Intervention Mapping. Second, we focus on the common thread that emerged through the steps to provide a comprehensive description of PMT programmes.

\section{Overview of the programmes}

\section{Step 1: Logic model of the problem}

Therapists perceived that anger and/or behaviour problems in their clients were associated with limitations in interoceptive awareness (IA; i.e., poor recognition and awareness of one's body signals) and limitations in adaptive coping skills(ACS); see Table 2.

\section{(insert Table 2)}

Therapists were asked whether anger and/or aggressive behaviour were the main reasons for referral for PTM. Only one therapist indicated that this is the case for a minority of his cases (40\%). For all other therapists more than half of their clients presented these problems (50-70\% of their caseload; $n=10)$, while for six this was the most common reason for client referral $(80-90 \%$ of their caseload). A lack of self-confidence $(n=10)$ and resilience $(n=9)$ are frequently (related) problems in individuals with MID-BIF that are grounds for referral to PMT.

\section{Step 2: Logic model of change}

The most frequently mentioned PMT objective was improving ACS. This included improving focussing on other or alternative behaviours (e.g., listening to music, stepping out of the situation), (physiological) arousal reduction through exercises (e.g., relaxation, breathing exercises), and improving ACS in social contexts (e.g., help seeking) when feeling angry or aroused. Another frequently mentioned goal in PMT is increasing IA. Overall IA (recognition and awareness of body signals not related to a specific emotion, which can be described as a perception of body signals) 
and/or anger related IA (recognition and awareness of body signals when angry, which is an interpretation of body signals) were mentioned as two specific components of IA (Table 3).

While in PMT programmes improving IA and ACS are the main objectives, other interventions have a strong focus on the external triggers leading to aggressive behaviour (e.g., cognitive behaviour therapy). The psychomotor therapists who were interviewed stated that, although working on these external triggers is important and should be part of the multidisciplinary approach, it is not a specific objective of PMT as they apply it (Table 3).

(insert Table 3)

\section{Step 3: Programme design}

Mechanisms of action of the PMT programme are explored in the third step of the Intervention Mapping. This step is divided in two sub steps. In the first sub step (step 3a), questions were asked about the theories / models linked to practical applications, whereas information on the methods linked to practical applications were asked in sub step $3 b$. Interviewees found this step challenging. In contrast to the information provided in the first two steps, several rounds of feedback and enquiry were needed to get the full picture of the theoretical base or models used.

Step 3a: Programme design Therapists mostly identified two major theoretical models underlying PMT for anger and aggressive behaviour in individuals with MID-BIF. Firstly, they referred to the model underlying cognitive behavioural therapy (CBT) that integrates traditional behavioural theories focussing on reinforcement mechanisms, social learning theory that adds observation as a key to learning and cognitive theory. Secondly, they referred to the General Aggression Model (GAM) as proposed by Anderson and Bushman (2002). GAM places similar emphasis on the environment and cognitions to account for learning processes. Two therapists reported that their frame of reference is the so-called Biopsychosocial Model as proposed by Engel (1980) who emphasised that behaviour and illness are affected by biological, psychological and sociological factors. In this model, the client's subjective experience is seen as essential and contributing to accurate diagnosis, health outcomes, and humane care (Borrell-Carrió, Suchman, \& Epstein, 2004). One therapist mentioned the humanistic theory, especially with emphasis on the unconditional positive regard (a concept developed by Rogers; Wilkins, 2000). 
The psychomotor therapists justified their choice of these theories and models by indicating these were proposed by literature and expert opinion. However, it should be mentioned that two psychomotor therapists indicated they did not use a theoretical model, rather were guided by their own clinical experience for the development and evaluation of their PMT programme.

Step 3b: Programme design Two PMT categories, namely action-oriented PMT and experienceoriented PMT, were used by the therapists (Probst et al., 2010). Action-oriented PMT focusses on learning by repeating or automatizing skills, whereas experience-oriented PMT focusses more on insight into emotions and the way of responding (caused by their emotional state). For this reason, experience-oriented PMT was used to improve IA, whereas action-oriented PMT was applied to increase the repertoire of ACS. The use of action-oriented and experience-oriented PMT was supported by the psychomotor therapists as PMT reflects the concrete learning style of individuals with MID-BIF and it is less dependent on their cognitive and verbal abilities.

The psychomotor therapists mentioned other methods: positive reinforcement and modelling. Furthermore, five psychomotor therapists noted that treatment involved working through the client's relapse prevention plan, practising and evaluating stressful situations and ways of coping.

Several practical PMT applications (activities as applied in PMT) were mentioned: (a) activities that emphasised IA, such as using tools to visualise tension (for example a tension thermometer ) and / or general exercises focussing on IA; (b) activities that aimed to improve ACS, such as arousal reduction techniques (e.g., progressive relaxation, applied relaxation and breathing exercises), focussing on other behaviour (e.g., stepping out of the situation), or activities that help practising new behaviour in social contexts (e.g., role plays). Also the connection between IA and ACS in activities was mentioned emphatically by several psychomotor therapists (such as by focussing on body signals while being challenged during a basketball exercise or home assignments focussing on reducing arousal when feeling tensed).

\section{Step 4: Programme production}

The PMT-programmes as described by the psychomotor therapists were embedded in a multidisciplinary treatment, as a complementary programme for anger regulation problems or 
aggressive behaviour. Fifteen psychomotor therapists suggested that they involved other people in the development of their PMT-programme. Table 4 shows the categories of other people as described by participants for each part of programme production.

(insert Table 4)

\section{Step 5: Implementation plan}

Few psychomotor therapists $(n=3)$ indicated they had direct responsibility for the implementation of the PMT-programme. All three mentioned limited time as the most important barrier for the implementation of a new programme. Half of the psychomotor therapists reported that it would be helpful to involve therapists from other disciplines, namely referrers $(n=8)$ and other experiential therapists (e.g., music therapy) $(n=3)$, in the context of referring to PMT.

Two psychomotor therapists invited people from the personal network of the client with MIDBIF (e.g., siblings or parents) to participate in a part of the PMT-programme. Where psychomotor therapists indicated that others (e.g. PMT-colleagues, staff or siblings) had been involved in delivering part of the PMT programme, it was also suggested that a manual of the programme was available ( $n=$ $5)$.

\section{Step 6: Evaluation plan}

Step 6a: Product evaluation Most psychomotor therapists suggested using an unstructured approach to outcome evaluation. This evaluation was mostly based on the views of the client and carers (e.g., parents) $(n=7)$ and / or on the views of the referrer $(n=3)$ regarding change in aggressive behaviour or ACS.

One interviewee did not answer this question. Only two psychomotor therapists described using specific outcome measures for IA (ABSQ; Anger Bodily Sensation Questionnaire, Zwets et al., 2014; or based on a self-constructed heart beat awareness test) or ACS (e.g., UCL; Utrecht Coping List [Utrechtse Coping Lijst], Schreurs, van de Willige, Brosschot, Tellegen, \& Graus, 1993). These two psychomotor therapists indicated that the instruments had not yet been validated in this population. Table 5 presents participants' responses on the product evaluation of PMT targeting anger and aggressive behaviour in individuals with MID-BIF.

(insert Table 5) 
Step 6b: Process evaluation Evaluation of the process of the treatment was done by seven psychomotor therapists, one of who did not provide a description of the type or content of the evaluation. The therapists evaluated the process together with others, by for example using feedback of the client $(n=3)$ or evaluating with colleagues $(n=3)$. In all but one cases, the process evaluation had led to changes in the programme $(n=5)$. Three psychomotor therapists planned to develop process evaluations in the future. Two therapists mentioned explicitly that the absence of guidelines to conduct a process evaluation is the main reason for not planning the evaluation.

\section{Outline of coherence of the programmes}

Table 6 presents the coherence between steps relating to the content of the applied programmes (steps 1, 2, 3 and 6). IA and ACS are the main elements of PMT that are mentioned throughout the different steps of the Intervention Mapping approach. Throughout the successive steps, the PMT-programmes seem coherent for ACS as every step builds on the previous steps. There appears to be less coherence across steps for IA. The use of the concepts 'overall IA' and 'anger related IA' in the first two steps was not consistent across therapists. Furthermore, the adoption of a theoretical orientation with regard IA was limited, while the effectiveness of IA was only seldom evaluated.

(insert Table 6)

\section{Conclusions}

The aim of this present study was to gather information on the theoretical orientation, content and evaluation of PMT as used with individuals with MID-BIF for anger regulation problems and / or aggressive behaviour. The Intervention Mapping approach offered a clear framework for mapping the results of the study. Findings suggested that anger regulation difficulties and aggressive behaviour were one of the main reasons for referral to PMT. There was general consensus amongst psychomotor therapists on the objectives of PMT as increasing IA and ACS. The Intervention Mapping approach also revealed that there were direct links between objectives (i.e., increasing IA and ACS) and the methods applied in the PMT programmes. Where ACS was the objective, there was a high level of PMT coherence with regard the theoretical orientation and evaluation methods. Where IA was the objective, the PMT application appeared less coherent: this was particularly prominent with regard the 
absence of a theoretical framework to account for the development of IA. In addition, therapists interviewed also indicated they did not have measures to assess clients' IA.

Where several treatment approaches (e.g., anger management) focus on ACS, in PMT this is combined with an extra focus on the body and the information the body offers (Zwets et al., 2016). This focus on IA makes PMT rather unique. In an experience oriented and action oriented approach, individuals are offered an environment with a focus on IA and ACS. Gratz and Roemer (2004) suggest that to prevent aggressive behaviour, individuals have to be able to respond with appropriate ACS when angry. This requires a broad repertoire of behavioural reactions. However, to act in an adaptive manner when angry, individuals also need to recognise this anger. IA that is the conscious perception of body signals related to anger (Farb et al., 2015; Füstös, Gramann, Herbert, \& Pollatos, 2012; Gratz \& Roemer, 2004; Mehling et al., 2012; Price \& Thompson, 2007). The PMT applications stress the link between IA and ACS, as both use information from the body to cope with anger, by using the body as a tool in therapy. For example, using "tension thermometers" participants are trained to timely pay attention to their emotional state, recognise what is happening and use this recognition as a starting point for selecting an appropriate reaction, thus preventing aggressive behaviour. PMT emphasises experiencing thoughts, feelings and behaviour. By creating a "playground", opportunities are provided to practise (new) ACS, such as ACS in social situations, using other behaviour or arousal reduction skills. The explicit focus on a possible lack of insight in one's own emotions and the fact that people can experience and train with old and new behaviour make these PMT applications suitable for individuals with MID-BIF.

The observation of gaps in the theoretical base and evaluation of IA is in line with the findings of our systematic review on PMT in this population, where evidence of the effectiveness of PMT was found specifically for ACS outcomes (Bellemans et al., in press). In this systematic review, IA was only implicitly targeted at in PMT interventions (e.g. meditation and relaxation). In the present study, the GAM was mentioned by several psychomotor therapists as reference framework for IA in PMT. The GAM provides a limited theoretical background for the use of IA. The GAM links arousal and affect in relation with aggressive behaviour. It remains unknown whether overall IA or anger related IA influence anger regulation when preventing aggressive behaviour. This can be seen in participants' 
inconsistent use of the terms 'overall IA' and 'anger related IA' in relation to determinants and specific outcomes on IA. The literature offers limited guidance on this point. Until now, only one study demonstrated that children with MID-BIF and aggressive behaviour have limited awareness of their body (Emck, Plouvier, \& van der Lee-Snel, 2012). Our results highlight the lack of clarity around IA, and its relation to aggressive behaviour. Similar to other target groups (Maas et al., 2015; Zwets et al., 2016) the effect of PMT on the IA skills of individuals with MID-BIF remains unclear. As stated by Cali and colleagues (2015) IA is shown to be a complex construct as it is closely related to interoceptive accuracy (the accuracy of one's own body signals), and emotional susceptibility (the tendency to experience feelings of discomfort when emotionally aroused) (Cali, Ambronsini, Piconi, Mehling and Committeri, 2015). How these concepts can be accurately measured is still a topic of debate in scientific research (Kanbara \& Fukunaga, 2016).

The absence of specific instruments for the evaluation of PMT - especially for anger related IA - is also in line with the literature. For example, the Anger Bodily Sensations Questionnaire (ABSQ; Zwets et al., 2014) is an instrument emphasising body awareness or IA, but has not yet been adapted and validated for use with individuals with MID-BIF. Moreover, none of the instruments for overall IA are adapted for use with individuals with MID-BIF although several new instruments have been developed to measure IA in a general population and psychiatric patients: e.g. the Mindfulness Attention Awareness Scale (MAAS; Brown \& Ryan, 2003), the Multidimensional Assessment of Interoceptive Awareness (MAIA; Mehling et al., 2012), the Scale of Body Connection (SBC; Price \& Thompson, 2007). In addition, an instrument measuring anger in association with ACS is also lacking. The Profile of Anger Coping Skills (PACS; Willner, Brace, \& Phillips, 2005) is an instrument to measure anger related coping skills in this population but it is not yet available in a Dutch translation.

The present findings have implications for the development and evaluation of PMT programmes for individuals with MID-BIF and aggressive behaviour. For the development of a standardized PMT programme finings highlighted a number of areas for improvement of PMT as an intervention. It is likely that a more crystallised description of the role of IA will positively influence the theoretical framework on which PMT is based on, and lead to a more sophisticated repertoire of practical PMT applications, thus increasing the effectiveness of PMT. Possibly the vasovagal theory of 
Porges (2009) will give handles for a stronger theoretical framework of PMT. Moreover, it is important to develop an adequate evaluation plan for PMT. However, to conduct a proper evaluation it is imperative to have measurable outcomes and thus specific instruments. Adaptations and/or translations of instruments for assessing IA (e.g., ABSQ) and ACS (e.g., PACS) are urgently needed. These instruments could be used as a standard practice within PMT to gather information on the mechanisms of action and the effectiveness of PMT.

The Intervention Mapping approach has shown to be a valuable tool to evaluate the process of the PMT-programme, revealing also the gaps in the coherence of the programme, an overview that was lacking until now. Intervention Mapping as used in this study can be used in the future as a tool for the process evaluation of PMT.

This study has several limitations that should be taken into account. First, the choice of Intervention Mapping over other tools (e.g., Online course on developing logic models and evaluation plans, Logic Model Overview) was based on the fact that Intervention Mapping allows both pinpointing and drawing up an inventory of PMT targeting anger regulation difficulties and aggressive behaviour in individuals with MID-BIF. Information provided by the psychomotor therapists reflects how PMT is currently applied by this group of therapists in Dutch residential facilities. However, we did not check the programmes in practice nor did we check its manuals, as these were generally not readily available. Second, it took several attempts for psychomotor therapists to formulate an answer, and in most cases they asked explicitly for examples to be able to answer the question. It is likely also that their responses might have been influenced by their education. It is not clear if and to what extent therapists' educational background has implications for their practice. Possibly psychomotor therapists apply these activities because they are educated to do so, even though strong theoretical foundation is lacking.

The present study has used a relatively novel approach to map the content and coherence of PMT programmes as currently applied in Dutch facilities serving clients with MID-BIF who present with anger problems and/or aggressive behaviour. This study may be a first step in developing evidence-based practice in which programme development, instrument validation and outcome 
14Assessing Psychomotor Therapy

evaluation are linked. In this way, PMT may add to the range of treatments that have been shown effective in reducing aggressive behaviour in clients with MID-BIF. Future research should focus on exploring the relationships between ADS, IA and anger. In particular, IA has seldom been examined in the scientific literature. IA is an interesting construct to explore and future research should aim at investigating the psychometric properties of the ABSQ for use with clients with MID-BIF and anger regulation difficulties.

\section{References}

Anderson, C. A., \& Bushman, B. J. (2002). Human aggression. Annual Review of Psychology, 53(1), $27-51$

Bellemans, T., Didden, R., Busschbach, J.T. van, Hoek, T.A.P., Scheffers, W.J., Lang, R.B., \& Lindsay, W.R. (In press). Psychomotor therapy targeting anger and aggressive behaviour in individuals with mild or borderline intellectual disabilities: a systematic review. Journal of Intellectual and Developmental Disability. doi:

$10.3109 / 13668250.2017 .1326590$

Boerhout, C., Swart, M., Busschbach, J.T. van, \& Hoek, H.W. (2016). Effect of aggression regulation on eating disorder pathology: RCT of a brief body and movement oriented intervention. European Eating Disorders Review, 24, 114-121.

Borrell-Carrió, F., Suchman, A. L., \& Epstein, R. M. (2004). The biopsychosocial model 25 years later: principles, practice, and scientific inquiry. Annals of Family Medicine, 2(6), 576-582.

Brown, K. W., \& Ryan, R. M. (2003). The benefits of being present: mindfulness and its role in psychological well-being. Journal of personality and social psychology, 84(4), 822-848.

Cali, G., Ambrosini, E., Picconi, L., Mehling, W.E., \& Committeri, G. (2015). Investigating the relationship between interoceptive accuracy, interoceptive awareness, and emotional susceptibility. Frontiers in Psychology, 6, 1202. doi: 10.3389/fpsyg.2015.01202

Chambless, D. L., \& Ollendick, T. H. (2001). Empirically supported psychological interventions: Controversies and evidence. Annual Review of Psychology, 52(1), 685-716. 
15Assessing Psychomotor Therapy

Eldredge, L.K.B., Markham, C.M., Ruiter, R.A.C., Fernándes, M.E., Kok, G., \& Parcel, G.S (2016). Planning health promotion programs: An intervention mapping approach ( $4^{\text {rd }}$ Ed.). Hoboken, NJ: John Wiley \& Sons.

Emck, C., Plouvier, M., \& van der Lee-Snel, M. (2012). Body experience in children with intellectual disabilities with and without externalising disorders. Body, Movement and Dance in Psychotherapy, 7(4), 263-275.

Engel, G.L. (1980). The clinical application of the biopsychosocial model. American Journal of Psychiatry, 137(5), 535-544.

Farb, N., Daubenmier, J., Price, C.J., Gard, T., Kerr, C., Dunn, B.D., Klein, A.C., Paulus, M.P., \& Mehling, W.E. (2015) Interoception, contemplative practice, and health. Frontiers in Psychology, 6, 763. doi: 10.3389/fpsyg.2015.00763

Füstös, J., Gramann, K., Herbert, B. M., \& Pollatos, O. (2013). On the embodiment of emotion regulation: interoceptive awareness facilitates reappraisal. Social Cognitive and Affective Neuroscience, 8(8), 911-917.

Gratz, K.L., \& Roemer, L. (2004). Multidimensional assessment of emotion regulation and dysregulation: Development, factor structure, and initial validation of the difficulties in emotion regulation scale. Journal of Psychopathology and Behavioral Assessment, 26(1), 41-54.

Goin-Kochel, R. P., Mackintosh, V. H., \& Myers, B. J. (2009). Parental reports on the efficacy of treatments and therapies for their children with autism spectrum disorders. Research in Autism Spectrum Disorders, 3, 528-537.

Goin-Kochel, R. P., Myers, B. J., \& Mackintosh, V. H. (2007). Parental reports on the use of treatments and therapies for children with autism spectrum disorders. Research in Autism Spectrum Disorders, 1, 195-209.

Kanbara, K. \& Fukunaga, M. (2016). Links among emotional awareness, somatic awareness and autonomic homeostatic processing. BioPsychoSocial Medicine, 10, 16. dio: 10.1186/s13030016-0059-3

Kay, J.J., Clegg, J.A., Emck, C., \& Standen, P.J. (2016). The feasibility of psychomotor therapy in acute mental health services for adults with intellectual disability. Journal of Intellectual and 
16Assessing Psychomotor Therapy

Developmental Disability, 41(1), 54-60.

Kok, G., Bartholomew, L. K., Parcel, G. S., Gottlieb, N. H., \& Fernández, M. E. (2014). Finding theory-and evidence-based alternatives to fear appeals: Intervention Mapping. International Journal of Psychology, 49(2), 98-107.

Maas, L.C.C. van der, Köke, A., Pont, M., Bosscher, R.J., Twisk, J.W.R., Janssen, T.W.J., \& Peters, M.L. (2015). Improving the multidisciplinary treatment of chronic pain by stimulating body awareness: A cluster-randomized trial. The Clinical Journal of Pain, 31(7), 660-669.

McDonnell, A., McCreadie, M., Mills, R., Deveau, R., Anker, R., \& Hayden, J. (2015). The role of physiological arousal in the management of challenging behaviours in individuals with autistic spectrum disorders. Research in Developmental Disabilities, 36, 311-322.

Mehling, W. E., Price, C., Daubenmier, J. J., Acree, M., Bartmess, E., \& Stewart, A. (2012). The multidimensional assessment of interoceptive awareness (MAIA). PloS One, 7(11), e48230.

Novaco, R. W. (1994). Anger as a risk factor for violence among the mentally disordered. In J. Monahan \& H. J. Steadman (Eds.), Violence and mental disorder: Developments in risk assessment (pp. 21-59). Chicago: University of Chicago Press.

Porges, S. W. (2009). The polyvagal theory: new insights into adaptive reactions of the autonomic nervous system. Cleveland Clinic Journal of Medicine, 76(Suppl 2), S86.

Price, C.J., \& Thompson, E.A. (2007). Measuring dimensions of body connection: Body awareness and bodily dissociation. Journal of Alternative and Complementary Medicine, 13, 945-953.

Probst, M., Knapen, J., Poot, G., \& Vancampfort, D. (2010). Psychomotor therapy and psychiatry: what's in a name. The Open Complementary Medicine Journal, 2(1), 105-113.

Röhricht, F. (2009). Body oriented psychotherapy. The state of the art in empirical research and evidence-based practice: a clinical perspective. Body, Movement and Dance in Psychotherapy, 4, 135-156.

Schaafsma, D., Stoffelen, J.M.T., Kok, G., \& Curfs, L.M.G. (2013). Exploring the development of existing sex education programs for people with intellectual disabilities: an intervention mapping approach. Journal of Applied Research in Intellectual Disabilities, 26, 157-166. 
17Assessing Psychomotor Therapy

Schreurs, P. J. G., Willige, G. van de, Brosschot, J. F., Tellegen, B., \& Graus, G. M. H. (1993). De Utrechtse Coping Lijst: UCL. Omgaan met problemen en gebeurtenissen [The Utrecht Coping List: UCL. Dealing with problems and incidents]. Lisse: Swets en Zeitlinger.

Singh, N.N. (Ed.) (2016). Handbook of evidence-based practices in intellectual and developmental disabilities. Cham: Springer International Publishing AG.

Sturmey, P., \& Didden, R. (Eds.) (2014). Evidence-based practice and intellectual disabilities. London: Wiley Blackwell.

Taylor, J. L., \& Novaco, R. W. (2005). Anger treatment for people with developmental disabilities: A theory, evidence and manual based approach. John Wiley \& Sons.

Wilkins, P. (2000). Unconditional positive regard reconsidered. British Journal of Guidance and Counselling, 28(1), 23-36.

Willner, P., Brace, N., \& Phillips, J. (2005). Assessment of anger coping skills in individuals with intellectual disabilities. Journal of Intellectual Disability Research, 49(5), 329-339.

Wieland, J., \& Zitman, F.G. (2015). It is time to bring borderline intellectual functioning back into the main fold of classification systems. British Journal of Psychiatry Bulletin, 40(4), 204-206.

Zwets, A.J., Hornsveld, R.H.J., Muris, P., Kanters, T., Langstraat, E., \& Marle, H.J.C. van (2016). Psychomotor therapy as an additive intervention for violent forensic psychiatric inpatients: a pilot study. International Journal of Forensic Mental Health, 15(3), 222-234.

Zwets, A.J., Hornsveld, R.H.J., Kraaimaat, F.W., Kanters, T., Muris, P., \& Marle, H.J.C. van (2014). The psychometric properties of the Anger Bodily Sensations Questionnaire (ABSQ). The Journal of Forensic Psychiatry \& Psychology, 25(4), 432-450. 